Draft version August 8, 2018

Typeset using LATEX twocolumn style in AASTeX61

\title{
EXTRAPLANAR H II REGIONS IN SPIRAL GALAXIES. II. IN SITU STAR FORMATION IN THE INTERSTELLAR THICK DISK OF NGC 4013
}

\author{
J. Christopher Howk,,${ }^{1,2}$ Katherine M. Rueff, ${ }^{1}$ Nicolas Lehner, ${ }^{1}$ Christopher B. Wotta, ${ }^{1}$ Kevin Croxall,${ }^{3,4}$ And \\ BLAIR D. SAVAGE ${ }^{5}$ \\ ${ }^{1}$ Department of Physics, University of Notre Dame, Notre Dame, IN 46556, USA \\ ${ }^{2}$ Instituto de Astrofísica, Pontificia Universidad Católica de Chile, Santiago, Chile \\ ${ }^{3}$ Department of Astronomy, The Ohio State University, Columbus, OH 43210, USA \\ ${ }^{4}$ Illumination Works LLC, 5550 Blazar Parkway \#150, Dublin, OH 43017, USA \\ ${ }^{5}$ Department of Astronomy, University of Wisconsin, Madison, Madison, WI 53706, USA
}

\section{ABSTRACT}

We present observations of an $\mathrm{H} \alpha$ emitting knot in the thick disk of NGC 4013, demonstrating it is an $\mathrm{H}$ II region surrounding a cluster of young hot stars $z=860 \mathrm{pc}$ above the plane of this edge-on spiral galaxy. With LBT/MODS spectroscopy we show this $\mathrm{H}$ II region has an $\mathrm{H} \alpha$ luminosity $\geq 4-7$ times that of the Orion nebula, with an implied ionizing photon production rate $\log Q_{0} \gtrsim 49.4$ (photons $\mathrm{s}^{-1}$ ). HST/WFPC2 imaging reveals an associated blue continuum source with $M_{V}=-8.21 \pm 0.24$. Together these properties demonstrate the H II region is powered by a young cluster of stars formed in situ in the thick disk with an ionizing photon flux equivalent to $\sim 6 \mathrm{O} 7 \mathrm{~V}$ stars. If we assume $\approx 6$ other extraplanar $\mathrm{H} \alpha$-emitting knots are $\mathrm{H}$ II regions, the total thick disk star formation rate of NGC 4013 is $\sim 5 \times 10^{-4} \mathrm{M}_{\odot} \mathrm{yr}^{-1}$. The star formation likely occurs in the dense clouds of the interstellar thick disk seen in optical images of dust extinction and $\mathrm{CO}$ emission.

Keywords: HII regions — ISM: abundances — galaxies: individual (NGC 4013) — galaxies: ISM — galaxies: spiral

\section{INTRODUCTION}

Stars form in cold, dense molecular clouds, from cores likely shaped by supersonic turbulence that collapse under the influence of gravity (McKee \& Ostriker 2007). The primary physical conditions needed for star formation are largely confined to the high-density regions in galactic disks. However, star formation has also been discovered in unusual locations throughout the universe, including in the far outer disks of galaxies (e.g., Ferguson et al. 1998; Werk et al. 2008; Thilker et al. 2007; Barnes et al. 2011), in tidally-stripped gas (e.g., de Mello et al. 2012; Stein et al. 2017; Urrutia-Viscarra et al. 2017), in ram-pressure stripped material (Cortese et al. 2004; Kenney et al. 2004), and even in a potentially-primordial ring of gas surrounding a group of galaxies (Thilker et al. 2009). These cases offer the opportunity to study the drivers of star formation in differing conditions.

Another extreme location for star formation is in the thick disks of spiral galaxies, where the pressures are much lower than in the midplane. Interstellar thick disks (within $\approx \pm 2 \mathrm{kpc}$, where the gas mosty corotates with the thin disk) are multiphase, including substantial quantities of dense or even molecular gas (García-Burillo et al. 1999; Howk \& Savage 1999, 2000). Candidate thick disk H II regions have been identified in a handful of galaxies as knots of extraplanar $\mathrm{H} \alpha$ emission (Walterbos 1991; Rand 1996; Howk \& Savage 2000; Rossa \& Dettmar 2000; Cortese et al. 2004; Rueff et al. 2013). Tüllmann et al. (2003) presented the first spectroscopic confirmation of two $\mathrm{H}$ II regions in the thick disk/halo of NGC $55(z \approx 1.1 \mathrm{kpc}, 2.2 \mathrm{kpc})$, which are consistent with being powered by single late-O/early-B stars.

This paper is the second in a series to study the implications of extraplanar H II regions in nearby edge-on galaxies. In the first of the series (Howk et al. 2018, hereafter Paper I) we considered the emission line spectrum from the brightest extraplanar H II region candidate identified by Rueff et al. (2013) at $z=860 \mathrm{pc}$ above the plane of NGC 4013 (at $[\alpha, \delta]_{\mathrm{J} 2000}=[11: 58: 33.2$, $+43: 57: 11.85]){ }^{1}$ We demonstrated that the knot of $\mathrm{H} \alpha$ emission was indeed an $\mathrm{H}$ II region associated with NGC 4013. This nebula, NGC 4013 EHR1 (EHR = extraplanar H II region following Tüllmann et al. 2003) is projected $R \approx 2.5 \mathrm{kpc}$ radially from the nucleus and

\footnotetext{
${ }^{1}$ We adopt $D=17.1 \pm 1.7 \mathrm{Mpc}$ to NGC 4013 (distance modulus of $31.17 \pm 0.10 \mathrm{mag}$ ), the mean distance to galaxy group 102 within which NGC 4013 resides (Tully et al. 2008).
} 


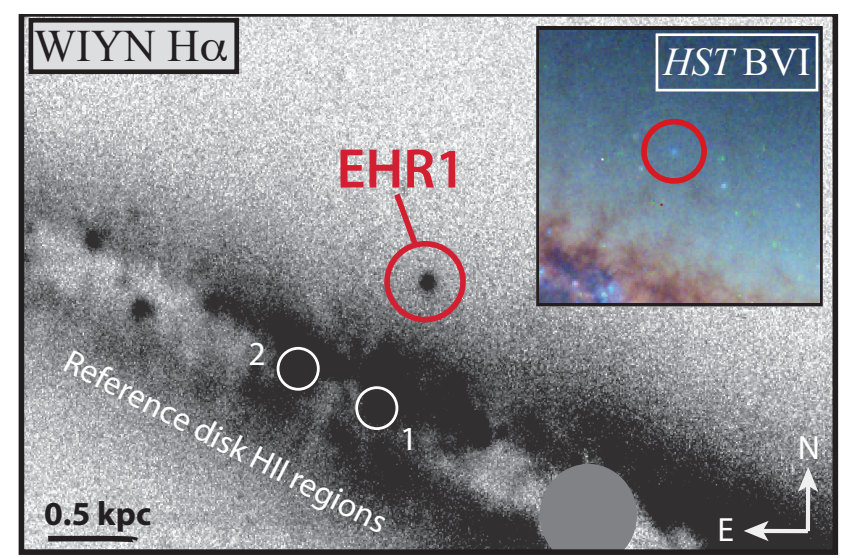

Figure 1. WIYN H $\alpha$ image (greyscale) and HST BVI image (three-color inset) of the brightest extraplanar H II region candidate in NGC 4013 (images described in Rueff et al. 2013). The extraplanar H II region NGC 4013 EHR1 lies at $z=860 \mathrm{pc}$ above the midplane. MODS longslit spectra reveal it is an $\mathrm{H}$ II region, while the $H S T$ photometry implies a cluster of young, O-type stars as the ionizing source for the nebula. Also marked are the two disk H II regions used to define the disk reference abundance (see Paper I). A bright foreground star is covered by a gray disk near the bottom of the image.

$z=860 \mathrm{pc}$ above the plane. We showed in Paper I that its metallicity is $\approx 1 / 2$ that of $\mathrm{H}$ II regions in the disk of NGC 4013 and discussed the implications of this low metallicity for flows through the thick interstellar disks of galaxies.

In this paper we focus on the nature of the stars that are powering the nebula NGC 4013 EHR1. Figure 1 shows WIYN $\mathrm{H} \alpha$ and Hubble Space Telescope $(H S T) /$ WFPC2 broad-band imagery (Rueff et al. 2013) of the nebula and its vicinity. The $H S T$ images show a blue continuum source coincident with the $\mathrm{H} \alpha$ emission. In what follows we demonstrate that the continuum emission is inconsistent with emission from a single star, implying it is a cluster, and that the evolutionary timescales imply the cluster was formed in situ in the thick disk. Our paper is arranged as follows. In $\S 2$ we briefly discuss the spectroscopy of this object and the implications for the underlying source that can be derived from the parameters of the $\mathrm{H}$ II region. In $\S 3$ we discuss the HST imaging of Rueff et al. (2013), deriving new photometric properties of the continuum emission associated with EHR1 and the origins of this emission. Lastly we discuss the implications of in situ star formation in the thick disks of galaxies in $\S 4$ and summarize our results in $\S 5$. EXTRAPLANAR NEBULA NGC 4013 EHR1

\subsection{LBT/MODS Observations}

We observed the $\mathrm{H} \alpha$ knot EHR1 using one side of the 8.4-m Large Binocular Telescope equipped with the first of the Multi-Object Double Spectrographs (MODS). ${ }^{2}$ We obtained $3 \times 1200$ sec longslit spectroscopy of NGC 4013 EHR1 using a 1".0 wide longslit with coverage $3,200 \lesssim \lambda \lesssim 10,000 \AA$. We use the MODS pipeline $^{3}$ to reduce the spectra, with details in Paper I.

Our final LBT/MODS spectrum is shown in Figure 2 , and a summary of derived properties important for the nature of the underlying stars is given in Table 1 . The emission line intensities are available in Paper I. In deriving the properties of the nebula EHR1 we have subtracted a background from the local stellar and diffuse ionized gas (DIG) emission. The DIG is particularly important, as it adds an emission line background with line ratios quite different than those of the nebula (Haffner et al. 2009). Our background spectrum is based on the geometric mean of regions immediately adjacent to either side of our extraction box for EHR1 (as we assume the emission falls off exponentially). We use a 1..5 extraction window, so that the DIG background is sampled 0 ". 75 to 2 .' 25 from the center of EHR1. Thus the DIG is sampled 36 to $100 \mathrm{pc}$ from the center of our target.

While the subtraction of the background DIG emission can have a sizeable impact on the line ratios used for estimating the metallicity, it has only a small impact on the diagnostics that impact our derived properties of the underlying stars (the subject of this paper). Our main diagnostics of the underlying stars are the Balmer lines, in which the DIG is significantly fainter than EHR1. Our DIG background spectrum has an $\mathrm{H} \alpha$ intensity that is only $\approx 13 \%$ of the emission from EHR 1 (whereas the intensities of the forbidden [N II] and [S II] are nearly equal from the DIG and EHR1). The errors in the $\mathrm{H} \alpha$ luminosity are more than twice this value (dominated by the extinction correction). Thus errors in the background spectrum would have to be much larger than expected to have significant impact on our total error budget.

The other main property derived from our spectroscopy that bears on the origin of the gas and stars is the velocity of the nebula EHR1 (Table 1). The appropriateness of that quantity depends on the nebula being placed accurately in the center of the slit. We positioned the slit using a blind offset from a nearby

${ }^{2}$ MODS is fully described in Pogge et al. (2010).
3 The MODS reduction pipeline was devel-
oped by K. Croxall with funding from NSF
Grant AST-1108693.
http://www.astronomy.ohio-state.edu/MODS/Software/modsIDL/.


bright star (with offsets derived from our WIYN imaging). The pointing of the instrument in these conditions should be accurate to a tenth of an arcsecond, implying a potential systematic error in the velocity of the H II region of up to $\approx 25 \mathrm{~km} \mathrm{~s}^{-1}$. Velocities derived for the DIG emission, which should largely fill the slit, should be limited only by the wavelength calibration of the spectrograph (which we confirm with sky absorption lines), though the intrinsic resolution is smaller given that it fills the slit. In the end we adopt an error of $\approx \pm 25 \mathrm{~km} \mathrm{~s}^{-1}$ for both.

\subsection{Characteristics of the Extraplanar H II Region}

The NGC 4013 EHR1 spectrum in Figure 2 is typical of a high-excitation H II region (see insets) and inconsistent with the thick disk DIG. The DIG near EHR1 has $[\mathrm{N} \mathrm{II}] / \mathrm{H} \alpha \approx 1$, whereas the nebula itself shows a ratio $\approx 0.1$.

We find a velocity $v_{\text {EHR } 1}=+720 \pm 25 \mathrm{~km} \mathrm{~s}^{-1}$, compared with a systemic velocity for NGC 4013 of $v_{\mathrm{sys}}=+835 \mathrm{~km} \mathrm{~s}^{-1}$ (Bottema 1995) (all heliocentric). The DIG (in our spectra), H I 21-cm emission (Zschaechner \& Rand 2015), and CO emission (García-Burillo et al. 1999) in this direction all show gas at velocities consistent with EHR1. The nebula is clearly associated with NGC 4013 and at velocities consistent with the local thick disk gas. The spectrum and velocity together demonstrate that NGC 4013 EHR1 is an H II region in the thick disk of NGC 4013.

Ideally one may be able to understand where EHR1 arises along the line of sight with enough information about the velocity structure of the local extraplanar material. The mean velocity of the DIG in our background spectra is $v_{\text {DIG }}=+680 \pm 25 \mathrm{~km} \mathrm{~s}^{-1}$, perhaps somewhat lower than EHR1. The local H I emission averaged over $\sim 1 \mathrm{kpc}$ is peaked at $v \approx+785 \mathrm{~km} \mathrm{~s}^{-1}$, with emission extending as low as $v \approx+650 \mathrm{~km} \mathrm{~s}^{-1}$ (Zschaechner \& Rand 2015). The brightest H I emission arises at lower $z$ than EHR1, though EHR1 lies well within the thick disk emission seen in the cubes of Zschaechner \& Rand. In practice, assessing EHR1's relationship to these ISM phases is complicated by the low velocity resolution of our DIG measurements and the low spatial resolution of the $\mathrm{H}$ I measurements.

In Paper I we found find a characteristic abundance $\epsilon(\mathrm{O}) \equiv 12+\log (\mathrm{O} / \mathrm{H})=8.35 \pm 0.15$ using the $\mathrm{N} 2[\equiv$ $I([\mathrm{~N} \mathrm{II}] 6584) / I(\mathrm{H} \alpha)]$ scale of Pettini \& Pagel (2004). The thick disk H II region has an abundance lower than the disk by $\Delta \epsilon(\mathrm{O})=-0.32 \pm 0.09$, i.e., by a factor of $\approx 2$ (Paper I). In this case, the comparison is made against the two H II regions in the disk identified as references in Figure 1. This discrepancy in metallicities of EHR1
Table 1. Properties of NGC 4013 EHR1

\begin{tabular}{|c|c|}
\hline Quantity & Value \\
\hline \multicolumn{2}{|c|}{ Stellar Properties } \\
\hline$L_{H_{\alpha}}\left(\operatorname{ergs~s}^{-1}\right)$ & $\geq(4.0 \pm 1.2) \times 10^{37 \mathrm{a}}$ \\
\hline $\log Q_{0}$ & $>49.3$ \\
\hline$M_{V}$ & $-8.21 \pm 0.24^{\mathrm{a}}$ \\
\hline$(B-V)_{0}$ & $-0.36 \pm 0.10$ \\
\hline$(V-I)_{0}$ & $+0.06 \pm 0.16$ \\
\hline \multicolumn{2}{|c|}{ H II Region Gas Properties } \\
\hline$v_{\text {helio }}\left(\mathrm{km} \mathrm{s}^{-1}\right)^{\mathrm{b}}$ & $+720 \pm 25$ \\
\hline$T_{e}(\mathrm{~K})$ & $\leq 13200$ \\
\hline$n_{e}\left(\mathrm{~cm}^{-3}\right)$ & $5-100$ \\
\hline $12+\log (\mathrm{O} / \mathrm{H})$ & $8.35 \pm 0.15$ \\
\hline Metallicity $\left(Z_{\odot}\right)^{\mathrm{c}}$ & $0.43 \pm 0.18$ \\
\hline \multicolumn{2}{|c|}{$\begin{array}{l}{ }^{a} \text { Assuming a distance of } 17.1 \pm 1.7 \mathrm{Mpc} \text { (Tully } \\
\text { et al. 2008) and an extinction given in the } \\
\text { text. Errors include a contribution from the } \\
\text { distance uncertainties where appropriate. }\end{array}$} \\
\hline \multicolumn{2}{|c|}{$\begin{array}{l}b \text { Based on a joint fit to the Balmer line ve- } \\
\text { locities. The forbidden metal lines yield a } \\
\text { similar velocity. }\end{array}$} \\
\hline \multicolumn{2}{|c|}{$\begin{array}{l}{ }^{c} \text { Assuming } 12+\log (\mathrm{O} / \mathrm{H}) \odot=8.72 \pm 0.03 \\
\text { (Steffen et al. } 2015) \text {. The more secure re- } \\
\text { sult is that NGC } 4013 \text { EHR1 has a gas-phase } \\
\text { oxygen abundance lower by }-0.32 \pm 0.09 \text { dex } \\
\text { than the disk of NGC } 4013 \text {. }\end{array}$} \\
\hline
\end{tabular}

compared with the disk implies a flow of metal-poor gas through the thick disk in this galaxy. It is unclear if this flow of matter is related to the formation of the cluster itself (we will need metallicity measurements of more extraplanar H II regions to demonstrate this).

The physical properties of the nebula are summarized in Table 1. The extinction-corrected $\mathrm{H} \alpha$ luminosity (see below) is $L_{\mathrm{H} \alpha} \approx(4.0 \pm 1.2) \times 10^{37} \mathrm{ergs} \mathrm{s}^{-1}$ (errors dominated by the extinction correction). In deriving this value, we apply corrections for slit losses, assuming a Gaussian image of $1^{\prime \prime}$ viewed through a perfect $1^{\prime \prime}$ slit, requiring a factor of 1.3 correction to our total fitted intensity. This value is $\geq(4-7) \times L_{\mathrm{H} \alpha}(\text { Orion })^{4}$ and requires an ionizing photon luminosity $\log Q_{0} \geq 49.4$ (Osterbrock \& Ferland 2006). This is equivalent to $\sim 6$ O7 V-equivalent stars (Martins et al. 2005). Although

\footnotetext{
4 The Orion nebula luminosity assumes a distance from Sandstrom et al. (2007) and integrated flux from Rumstay (1984).
} 


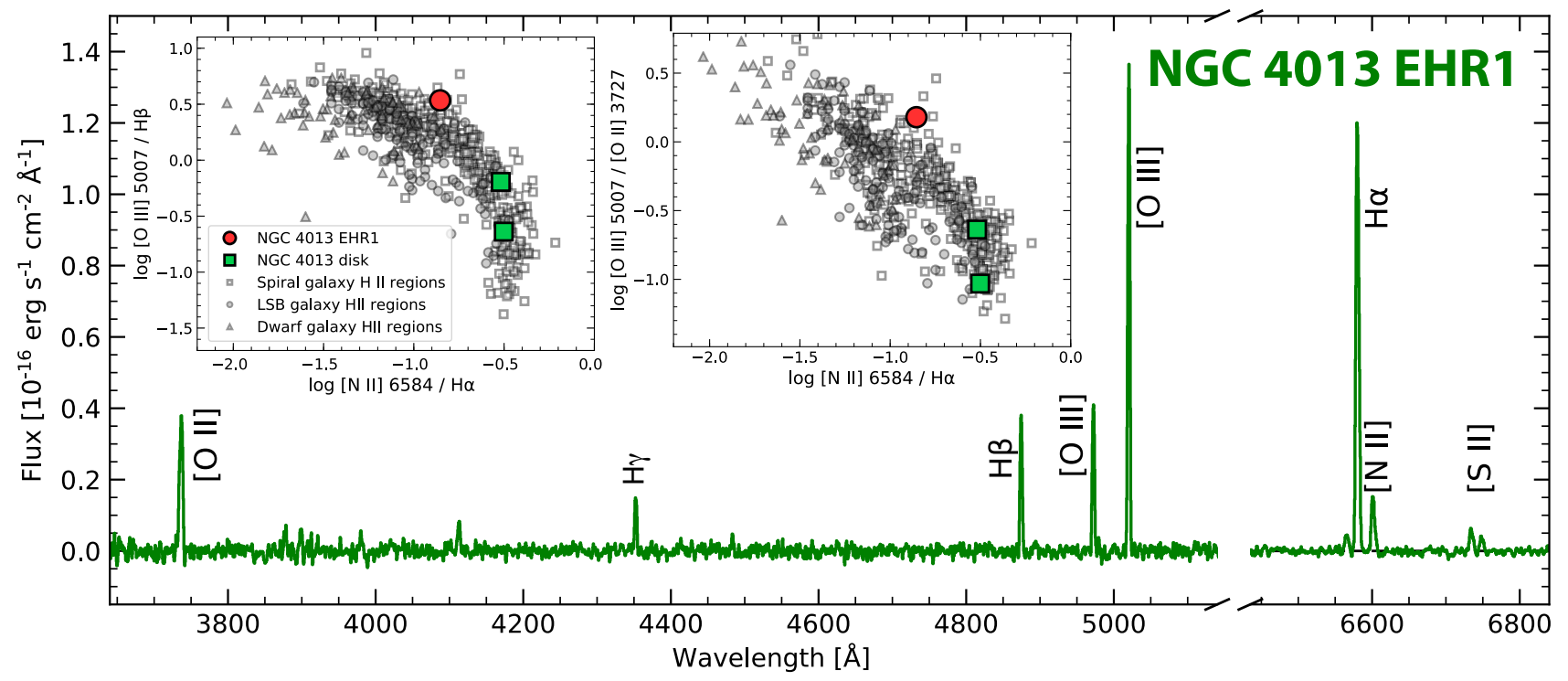

Figure 2. LBT/MODS spectrum of NGC 4013 EHR1. Several hydrogen Balmer lines and collisionally-excited metal lines are shown, notably those important for assessing the gas-phase abundance. Inset - Characteristic emission line diagnostic ratios for NGC 4013 EHR1 compared with H II regions in the disks of spiral, low surface brightness, and dwarf galaxies in the local universe (Berg et al. 2015; Croxall et al. 2015; Croxall et al. 2016; Bresolin \& Kennicutt 2015; van Zee \& Haynes 2006). EHR1 has emission line ratios consistent with those seen in other high-excitation H II regions in the local universe.

the $\mathrm{H} \alpha$ luminosity could nearly be met by a single $\mathrm{O} 4 \mathrm{~V}$ star, we rule this out in $\S 3$.

\section{THE ORIGINS OF THE STELLAR CLUSTER ASSOCIATED WITH NGC 4013 EHR1}

\subsection{HST/ WFPC2 Imaging of the Continuum Source}

The central cluster of NGC 4013 EHR1 is detected in the three-filter (F435W/F555W/F814W) HST/WFPC2 images of Rueff et al. (2013), as shown in Figure 1. We derive Johnson-Cousins magnitudes from aperture photometry $\left\{B, V, I_{C}\right\}_{V e g a}=\{23.51 \pm 0.04,23.65 \pm$ $0.06,23.24 \pm 0.11\}^{5}$ Our Balmer line analysis implies $E(B-V)=0.22 \pm 0.07$ - equivalent to $A_{V}=0.69 \pm 0.21$ and $E(V-I)=0.35 \pm 0.11$ assuming a Cardelli et al. (1989) extinction curve and $R_{V}=3.1$. The foreground extinction due to the Milky Way in this direction contributes $A_{V} \approx 0.05$ mag of the total (Schlafly \& Finkbeiner 2011); thus most of the extinction arises within NGC 4013 itself. Together our photometry and extinction estimates yield the intrisic colors and absolute magnitude of the central source summarized in Table 1.

The WFPC2 images demonstrate the continuum source underlying the extraplanar H II region is partly-

\footnotetext{
${ }^{5}$ We derive filter transformations assuming a mid-O star spectral type calculated with the STSDAS routine calcphot. The filter transformations do not vary strongly with OB-star subtype $(<0.02 \mathrm{mag})$.
}

resolved and thus not a single star. Figure 3 shows the photometric curve of growth (the amount of the light enclosed within varying sized apertures) for the F555W (V-band) images of NGC 4013 EHR1 (blue) compared with the mean behavior of three likely point sources (thick black line). For each source, we normalize the curves to their total integrated flux within $1^{\prime \prime} 0$ (with sky subtraction performed locally based on an annulus with inner and outer radii $1^{\prime \prime}\left(0\right.$ and $\left.1^{\prime \prime} .5\right)$. The grey shaded area shows the extreme values from our reference objects at each aperture size, with the curves for the individual objects shown as thin grey lines. Our reference sources were chosen to be likely point sources: identifying bona fide point sources in the small WFPC2 field of view - which is dominated by NGC 4013 - is difficult. It is made even more difficult by the presence of clusters associated with NGC 4013 itself. We vetted potential reference point sources initially through visual inspection to rule out partially resolved galaxies. We fit our final candidates with a two-dimensional Gaussian (though a Moffat function fits as well), and we found the residuals for these three are largely symmetrical (the fit gives no evidence for any ellipticity). All four reference sources are in very good agreement to $\sim 75 \%$ of the encircled light, thus the core of the point spread function traced by these objects is well characterized through the mean curve in Figure 3. It is clear from Figure 3 that EHR1 is more extended than these sources. 


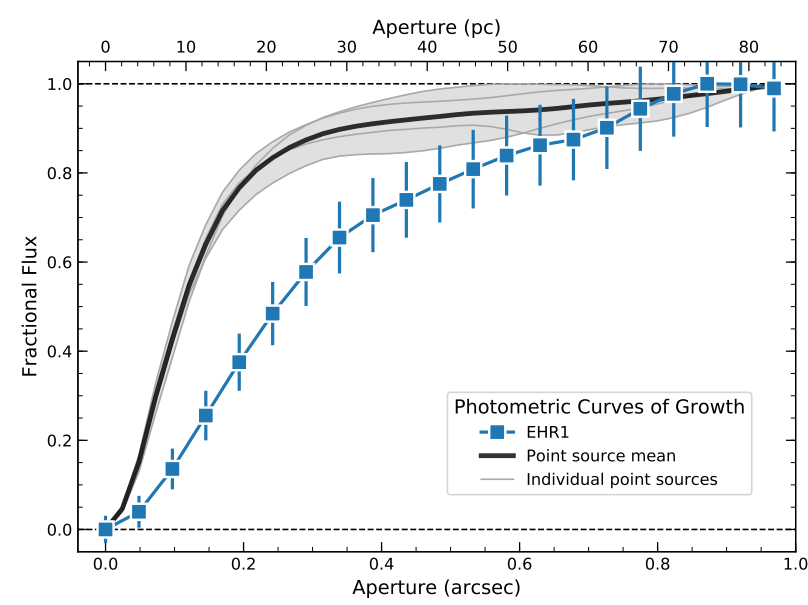

Figure 3. The photometric curve of growth of EHR1 (blue) compared with the mean behavior of three reference point sources (black) showing the fraction of the integrated flux contained within apertures of differing sizes. The aperture radii are given in arcseconds along the bottom and parsecs along the top of the plot. The shaded regions show the extrema from the three reference sources at each aperture size, while the curves for the individual reference objects are shown as thin grey lines. The light from EHR1 is clearly more extended than for the reference sources. The uncorrected half-light radius of EHR 1 is $\sim 0$.' 25 or $\sim 22$ pc. Assuming the mean profile of the reference sources is appropriate for deconvolving the EHR1 data, the intrinsic half-light radius is $\sim 0$.' 22 or $\sim 19$ pc.

The continuum source has a (deconvolved) FWHM $\approx$ $12 \mathrm{pc}\left(0^{\prime \prime} 144\right)$ and half-light radius $\approx 19 \mathrm{pc}\left(0^{\prime \prime} 22\right)$ in the F555W images. The FWHM values are based on twodimensional Gaussian fits to the light profiles. We use the mean FWHM of fits to the reference point sources to deconvolve the EHR1 fits. This size is on the large end - but consistent with - those of blue clusters found in nearby galaxies (e.g., Larsen 1999).

\subsection{A Thick Disk Star Cluster Formed in situ}

Our HST/WFPC2 images demonstrate NGC 4013 EHR1 hosts a cluster of stars at $z=860 \mathrm{pc}$ above the plane. In addition to being resolved, the source is too bright to be a single star. We find $M_{V}=-8.21 \pm 0.24$, which is 2.7 and 3.6 magnitudes brighter than single O4 V or O7 V stars, respectively (Martins et al. 2005). The optical output is thus equivalent to $\sim 10 \mathrm{O} 4 \mathrm{~V}$ or 30 O7 V stars. The continuum emission is $\sim 2$ magnitudes $(\sim 5 \times)$ brighter than even the brightest individual supergiants (Martins et al. 2005). Thus, the photometric properties of the source indicate the underlying continuum source must represent many $\mathrm{O}$ stars. Following the Appendix of Werk et al. (2008), this number of O7 V-equivalent stars implies the presence of 5-200 O-type stars (of all subtypes; this calculation depends on the highest-mass star present, which is unknown). The ionizing photon production powering the $\mathrm{H} \alpha$ emission is dominated by the earliest spectral types, while the optical output has a larger contribution from later spectral types. It could be possible that some of the ionizing radiation is leaking from the $\mathrm{H}$ II region into the neighboring DIG, given the larger requirements of the optical emission compared with the $\mathrm{H} \alpha$ luminosity. However, this simple comparison does not completely account for the full optical contributions from all the stars likely to be present (an accounting that is made difficult by stochastic sampling of the initial mass function in this relatively low-mass cluster).

The intrinsic colors for the stellar cluster associated with NGC 4013 EHR1 imply the optical emission is dominated by $\mathrm{O}$ stars, as the colors are consistent with the degenerate colors produced by these massive stars (Martins \& Plez 2006). If the light from this object were dominated by an individual star, the colors limit the age to $\tau \lesssim 3-10 \mathrm{Myr}$ (Schaller et al. 1992). For the integrated light from a cluster, the $(B-V)_{0}$ and $(V-I)_{0}$ colors are indicative of ages $\tau \lesssim 6 \mathrm{Myr}$ (Pandey et al. 2010). If this cluster was ejected after forming in the disk of the galaxy, reaching its observed height in this time would require a vertical velocity of $v_{z} \gtrsim 100-300$ $\mathrm{km} \mathrm{s}^{-1}$. This is an extremely high peculiar velocity for a cluster of stars. Even if one could imagine a mechanism for providing this vertical kick to an entire cluster of stars, if the vertical velocity is added to an assumed azimuthal velocity due to rotation, the total cluster speed would approach or exceed the escape speed of the galaxy.

It could be postulated that this thick disk cluster and nebula in NGC 4013 are associated with a disrupted dwarf galaxy. However, this is extremely unlikely, as the accreted cluster would have to be deposited into the thick disk at an extremely small - and statisticallyunlikely - distance from the center of NGC 4013 (the "bulls-eye" problem of Peek 2009). Though there is evidence for a major merger $3-5$ Gyr ago (Wang et al. 2015) based on NGC 4013's prodigious warp (Bottema 1996; Zschaechner \& Rand 2015) and recentlydiscovered stellar stream (Martínez-Delgado et al. 2009), the very young age of the stars in this cluster are inconsistent with the timescales of that event.

Thus, we conclude NGC 4013 EHR1 is powered by a cluster of young, hot stars that formed in situ within the thick disk of NGC 4013.

\section{THICK DISK STAR FORMATION}

NGC 4013 EHR1 is a thick disk H II region located at $z=860 \mathrm{pc}$ above the plane of the edge-on spiral 
galaxy NGC 4013. It is powered by a young stellar cluster that, based on timing constraints, formed in situ in the thick disk. The properties of this thick disk H II region and its stars demonstrate that: a) the thick disk contains at least some dense, even molecular gas from which the stars formed; b) star formation can proceed in the relatively low-pressure environments of the thick disk; and c) the origins of the gas in the thick disk are highly varied, including material expelled from the thin disk as well as metal poor gas originating in the corona or beyond (as discussed in Paper I).

The formation of hot, massive stars requires a substantial mass of dense, cold (likely molecular) clouds. While it is often assumed that such cold gas is confined to the thin disk, there is strong evidence for its presence in the thick disks of spiral galaxies. García-Burillo et al. (1999) detected extraplanar CO filaments to $z \lesssim 300 \mathrm{pc}$ above the disk of NGC 4013, demonstrating the presence of $\mathrm{H}_{2}$-bearing gas several scale heights above the plane (with a total mass of order $\sim 10^{7} \mathrm{M}_{\odot}$ ). The extraplanar $\mathrm{CO}$ may not be wide-spread, as it was only imaged over the central regions of the galaxy. Some of the CO may in fact be associated with a large $\mathrm{H} \alpha$ structure tracing an apparent outflow from a nuclear starburst (Rand 1996).

While some of the $\mathrm{CO}$ emission could be associated with rather specific structures associated with nuclear outflows, we also see dusty thick disk clouds visible in absorption against the background stellar light over a significant fraction of the disk. Such clouds are common to spiral galaxies (Howk \& Savage 1999, 2000). These clouds are only visible due the contrast they produce, requiring them to be denser than their surroundings. Their high densities $\left(n_{\mathrm{H}} \gtrsim 10\right.$ to $\left.\gtrsim 25 \mathrm{~cm}^{-3}\right)$ and high H I columns $\left(\log N(\mathrm{H} \mathrm{I}) \gtrsim 10^{20}-10^{21}\right)$ are consistent with molecular-bearing clouds in the Milky Way, and the cloud masses approach those of giant molecular clouds (Howk \& Savage 2000; Rueff et al. 2013).

Thus, the fuel for star formation exists in the thick disk of NGC 4013, but why does such dense, cold gas exist in the thick disk? The vast majority of the gas in the thick disk is thought to have been ejected from the plane, e.g., by the effects of stellar feedback (Norman \& Ikeuchi 1989) or through hydrodynamic effects (see Howk \& Savage 1997, for an extensive discussion). Indeed, the dust seen in broadband optical images (such as the inset of Figure 1) almost certainly originated in the thin disk, as the dust content of infalling or condensing matter is typically low. It may be the case that the material is lifted from the disk cold, either as the walls of expanding shells or as material entrained in the outflows. While it seems likely the transfer of momentum from the hot fluid to the cold clouds would be inefficient, molecular gas is seen in outflows from starbursts (e.g., Walter et al. 2017).

Even if the gas lifted from the disk is not originally cold/dense enough to support star formation, the interactions of adjacent outflows in the thick disk can serve to produce cold material. Converging flows of neutral material can compress gas and induce cooling and star formation (e.g., Heitsch et al. 2008; Wu et al. 2017); the convergence of the walls of adjacent supershells in the thick disk may be a trigger for cloud formation (Ntormousi et al. 2011). Indeed, the propensity for outflows to cause the compression of material leading to cold cloud formation was noted some time ago, whether the outflows were driven by feedback (de Avillez 2000) or by hydraulic jumps at spiral arm shocks (Martos et al. 1999). Gravitational effects, such as tidal perturbations by passing globular clusters (de la Fuente Marcos \& de la Fuente Marcos 2008) or extended density waves (Struck \& Smith 2009), could also plausibly lead to triggering cold cloud formation and star formation in high-altitude gas.

While there is extraplanar star formation in NGC 4013, it is not so substantial that it will strongly affect the evolution of the thick disk. If we assume all the $\mathrm{H} \mathrm{II}$ region candidates trace star formation (6 EHRs in total with $0.86 \lesssim z \lesssim 3.4 \mathrm{kpc}$ ), the total $\mathrm{H} \alpha$ luminosity of these candidate $\mathrm{H}$ II regions is $L_{\mathrm{H} \alpha \text {,total }} \sim 7 \times 10^{37}$ $\operatorname{ergs~s}^{-1}$ (using the MODS spectra of EHR1 to calibrate our $\mathrm{H} \alpha$ images). This implies a total thick disk star formation rate SFR $\sim 5 \times 10^{-4} \mathrm{M}_{\odot} \mathrm{yr}^{-1}$ in NGC 4013 (using the $\mathrm{H} \alpha$-SFR calibration of Kennicutt \& Evans 2012).

This star formation is also not likely to strongly impact the interstellar thick disk. One could imagine the presence of young, hot stars in the thick disk could contribute to the ionization of the DIG, which otherwise requires the escape of significant ionizing flux from the disk (Haffner et al. 2009). If photons produced by in situ thick disk stars experience significantly lower opacity, they could contribute disproportionately to powering the DIG. However, the ionizing photon budgets typically required for the DIG are typically $\gtrsim 10 \%$ of that produced in the thin disk (Haffner et al. 2009), several orders of magnitude higher than that available from the thick disk star formation in NGC 4013.

The star forming environments of the thick disk may be similar to those encountered in the outskirts of galaxies (e.g., Ferguson et al. 1998) or in tidal matter, including the tidal dwarf galaxies (e.g., Werk et al. 2008; de Mello et al. 2012). In these cases, the average gas densities and pressures are expected to be lower than in the mid-plane of a massive spiral. Additionally, as is the 
case with tidally-stripped material, there is not a consistent gravity vector drawing the clouds together. In order to reach the high densities needed for star formation, some combination of spontaneous thermal instabilities, tidal forces (for stripped gas), or converging flows may be required. As more information becomes available on extraplanar H II regions, a comparison of the SFRs and $\mathrm{H} \mathrm{I+CO}$ columns should allow us to assess global star formation scaling laws at the lowest SFRs.

There are now a few extraplanar H II regions for which spectroscopic confirmation has been presented in the literature (Tüllmann et al. 2003; Stein et al. 2017, Paper I). We summarize their properties in Table 3. The H II regions in Stein et al. (2017) are associated with tidallyand ram pressure-stripped gas about spiral galaxies, reminiscent of those studied in tidal debris by Werk et al. (2008). Two of their three H II regions show disk-like metallicities, a third is marginally lower. In NGC 55 (Tüllmann et al. 2003), the extraplanar H II regions found at $z \sim 1.1$ and $2.2 \mathrm{kpc}$ have abundances higher than the local disk (in the reassessment of $\mathrm{Pa}$ per I). This dwarf galaxy shows an incredible wealth of H $\alpha$ filaments and loops (Ferguson et al. 1996; Tüllmann et al. 2003), and the H II regions may probe gas associated with large-scale outflows from the disk. The extraplanar H II regions in NGC 4013 and NGC 55 at the very least imply metallicities of the thick disk are inhomogeneous on relatively small scales. Future observations will help us assess wehther these changes represent organized gradients as opposed to stochastic variations within the complex distribution of gas and dust in the thick disk of this and other galaxies.

The extraplanar H II regions so far reported in the luminous spiral galaxies all require several O-type stars to provide for their ionization, although there is almost certainly a selection effect at work given the small number of $\mathrm{H}$ II regions so far described in the literature. NGC 4013 EHR1 has a luminosity as large as those H II regions forming in tidal debris about NGC 3628 and NGC 4522, even though the mechanisms for forming the clusters powering these $\mathrm{H}$ II regions are likely quite different. The luminosity of NGC $55 \mathrm{EHR}^{6}$ is nearly an order of magnitude lower than those for the brightest in the more luminous spiral galaxies (Ferguson et al. 1996). This H II region is consistent with being powered by a single O9.5 or B0 star (Stein et al. 2017).

\section{SUMMARY}

\footnotetext{
${ }^{6}$ Only the luminosity for NGC 55 EHR2 was reported in Ferguson et al. (1996), which is the value used by Stein et al. (2017).
}

We have presented observations of a region of the thick disk in NGC 4013 that shows strong, point-like $\mathrm{H} \alpha$ emission and a blue continuum source. Based on ground-based optical spectroscopy and HST imaging, we demonstrate:

1. The source EHR1 represents an H II region projected $z=860 \mathrm{pc}$ above the plane of this galaxy. The ionizing flux required by the $\mathrm{H} \alpha$ emission is $\approx 4-7$ times that of the Orion Nebula, requiring the equivalent of $\sim 6 \mathrm{O} 7 \mathrm{~V}$ stars.

2. The optical emission from the underlying continuum source is slightly extended, with a $\mathrm{FWHM} \approx$ $12 \mathrm{pc}$. Thus the object appears to be a cluster of stars in the thick disk.

3. The absolute magnitude and colors of the emission require contributions from young stars, the equivalent of $\sim 30 \mathrm{O} 7 \mathrm{~V}$ stars. The timescales for the evolution of $\mathrm{O}$ stars are inconsistent with the cluster's formation in the disk. Thus the star were formed in situ in the thick disk.

4. EHR1 is by far the $\mathrm{H} \alpha$-brightest of the extraplanar H II region candidates in NGC 4013. The total star formation rate of the thick disk in this galaxy is quite low, $\sim 5 \times 10^{-4} \mathrm{M}_{\odot} \mathrm{yr}^{-1}$. While such a low rate of star formation in spiral galaxies will not change the appearance of their thick disk populations, its presence allows us to probe the metallicity of thick disk gas and to study star formation in unusual environments.

JCH recognizes the hospitality of the Instituto de Astrofísica, Pontificia Universidad Católica de Chile during the writing of this work. We acknowledge support from NASA through grant NNX10AE87G. Based on data acquired using the Large Binocular Telescope (LBT). The LBT is an international collaboration among institutions in the US, Italy, and Germany. LBT Corporation partners are the University of Arizona, on behalf of the Arizona university system; Instituto Nazionale do Astrofisica, Italy; LBT Beteiligungsgesellschaft, Germany, representing the Max Planck Society, the Astrophysical Institute of Postdam, and Heidelberg University; Ohio State University, and the Research Corporation, on behalf of the University of Notre Dame, the University of Minnesota, and the University of Virginia. Includes observations obtained with the NASA/ESA Hubble Space Telescope operated at the Space Telescope Science Institute, which is operated by the Association of Universities 
Table 3. Extraplanar H II Region Properties

\begin{tabular}{lccccc}
\hline \hline \multicolumn{1}{c}{ Galaxy } & Nebula ID & $z(\mathrm{pc})$ & $\Delta \epsilon(\mathrm{O})$ & $L_{\mathrm{H} \alpha}\left(\mathrm{ergs} \mathrm{s}^{-1}\right)$ & Reference \\
\hline NGC 4013 & 1 & 0.9 & $-0.32 \pm 0.09$ & $4 \times 10^{37}$ & This work \\
NGC 55 & 1 & 1.1 & $+0.24 \pm 0.12$ & $\ldots$ & Tüllmann et al. (2003) \\
NGC 55 & 2 & 2.2 & $+0.29 \pm 0.12$ & $6 \times 10^{36}$ & Tüllmann et al. (2003) \\
NGC 3628 & 2 & 2.8 & $-0.17 \pm 0.10$ & $2 \times 10^{37}$ & Stein et al. (2017) \\
NGC 3628 & 3 & 3.0 & $-0.05 \pm 0.11$ & $1 \times 10^{37}$ & Stein et al. (2017) \\
NGC 4522 & 1 & 1.4 & $-0.03 \pm 0.07$ & $6 \times 10^{37}$ & Stein et al. (2017) \\
\hline
\end{tabular}

Note-The nebular IDs are those reported in the original references. All abundance offsets $\Delta \epsilon(\mathrm{O})$ were derived anew in Paper I using the intensities reported in the original references. Where more than one disk reference region is available (for NGC 4013 and NGC 4522), the values for $\Delta \epsilon(\mathrm{O})$ represent the joint distribution. The $\mathrm{H} \alpha$ luminosity for NGC 55 EHR2 quoted in Stein et al. (2017) comes from the images of Ferguson et al. (1996). We have corrected the values used in those works to reflect the new distance $D=2.3 \mathrm{Mpc}$ from Kudritzki et al. (2016).

for Research in Astronomy, Inc., under NASA contract NAS5-26555.

Facilities: LBT(MODS), HST(WFPC2), WIYN

Software: Astropy (Price-Whelan et al. 2018), Matplotlib (Hunter 2007)

\section{REFERENCES}

Barnes, K. L., van Zee, L., \& Skillman, E. D. 2011, ApJ, 743,137

Berg, D. A., Skillman, E. D., Croxall, K. V., et al. 2015, ApJ, 806, 16

Bottema, R. 1995, A\&A, 295, 605

-. 1996, A\&A, 306, 345

Bresolin, F., \& Kennicutt, R. C. 2015, MNRAS, 454, 3664

Cardelli, J. A., Clayton, G. C., \& Mathis, J. S. 1989, ApJ, 345,245

Cortese, L., Gavazzi, G., Boselli, A., \& Iglesias-Paramo, J. 2004, A\&A, 416, 119

Croxall, K. V., Pogge, R. W., Berg, D. A., Skillman, E. D., \& Moustakas, J. 2015, ApJ, 808, 42

Croxall, K. V., Pogge, R. W., Berg, D. A., Skillman, E. D., \& Moustakas, J. 2016, ApJ, 830, 4

de Avillez, M. A. 2000, MNRAS, 315, 479

de la Fuente Marcos, R., \& de la Fuente Marcos, C. 2008, ApJL, 685, L125

de Mello, D. F., Urrutia-Viscarra, F., Mendes de Oliveira, C., et al. 2012, MNRAS, 426, 2441

Ferguson, A. M. N., Wyse, R. F. G., \& Gallagher, J. S. 1996, AJ, 112, 2567

Ferguson, A. M. N., Wyse, R. F. G., Gallagher, J. S., \& Hunter, D. A. 1998, ApJL, 506, L19
García-Burillo, S., Combes, F., \& Neri, R. 1999, A\&A, 343, 740

Haffner, L. M., Dettmar, R.-J., Beckman, J. E., et al. 2009, Reviews of Modern Physics, 81, 969

Heitsch, F., Hartmann, L. W., Slyz, A. D., Devriendt, J. E. G., \& Burkert, A. 2008, ApJ, 674, 316

Howk, J. C., Rueff, K. M., Lehner, N., et al. 2018, ApJ, submitted (Paper I)

Howk, J. C., \& Savage, B. D. 1997, AJ, 114, 2463

—. 1999, AJ, 117, 2077

—. 2000, AJ, 119, 644

Hunter, J. D. 2007, Computing In Science \& Engineering, 9,90

Kenney, J. D. P., van Gorkom, J. H., \& Vollmer, B. 2004, AJ, 127, 3361

Kennicutt, R. C., \& Evans, N. J. 2012, ARA\&A, 50, 531

Kudritzki, R. P., Castro, N., Urbaneja, M. A., et al. 2016, ApJ, 829, 70

Larsen, S. S. 1999, A\&AS, 139, 393

Martínez-Delgado, D., Pohlen, M., Gabany, R. J., et al. 2009, ApJ, 692, 955

Martins, F., \& Plez, B. 2006, A\&A, 457, 637

Martins, F., Schaerer, D., \& Hillier, D. J. 2005, A\&A, 436, 1049 
Martos, M., Allen, C., Franco, J., \& Kurtz, S. 1999, ApJL, 526, L89

McKee, C. F., \& Ostriker, E. C. 2007, ARA\&A, 45, 565

Norman, C. A., \& Ikeuchi, S. 1989, ApJ, 345, 372

Ntormousi, E., Burkert, A., Fierlinger, K., \& Heitsch, F. 2011, ApJ, 731, 13

Osterbrock, D. E., \& Ferland, G. J. 2006, Astrophysics of gaseous nebulae and active galactic nuclei

Pandey, A. K., Sandhu, T. S., Sagar, R., \& Battinelli, P. 2010, MNRAS, 403, 1491

Peek, J. E. G. 2009, ApJ, 698, 1429

Pettini, M., \& Pagel, B. E. J. 2004, MNRAS, 348, L59

Pogge, R. W., Atwood, B., Brewer, D. F., et al. 2010, in Proc. SPIE, Vol. 7735, Ground-based and Airborne Instrumentation for Astronomy III, 77350A

Price-Whelan, A. M., Sipőcz, B. M., Günther, H. M., et al. 2018, ArXiv e-prints, arXiv:1801.02634

Rand, R. J. 1996, ApJ, 462, 712

Rossa, J., \& Dettmar, R.-J. 2000, A\&A, 359, 433

Rueff, K. M., Howk, J. C., Pitterle, M., et al. 2013, AJ, 145,62

Rumstay, K. S. 1984, PhD thesis, Ohio State University, Columbus.

Sandstrom, K. M., Peek, J. E. G., Bower, G. C., Bolatto, A. D., \& Plambeck, R. L. 2007, ApJ, 667, 1161

Schaller, G., Schaerer, D., Meynet, G., \& Maeder, A. 1992, A\&AS, 96, 269

Schlafly, E. F., \& Finkbeiner, D. P. 2011, ApJ, 737, 103
Steffen, M., Prakapavičius, D., Caffau, E., et al. 2015, A\&A, 583, A57

Stein, Y., Bomans, D. J., Ferguson, A. M. N., \& Dettmar, R.-J. 2017, A\&A, 605, A5

Struck, C., \& Smith, D. C. 2009, MNRAS, 398, 1069

Thilker, D. A., Bianchi, L., Meurer, G., et al. 2007, ApJS, 173,538

Thilker, D. A., Donovan, J., Schiminovich, D., et al. 2009, Nature, 457, 990

Tüllmann, R., Rosa, M. R., Elwert, T., et al. 2003, A\&A, 412,69

Tully, R. B., Shaya, E. J., Karachentsev, I. D., et al. 2008, ApJ, 676, 184

Urrutia-Viscarra, F., Torres-Flores, S., Mendes de Oliveira, C., et al. 2017, ArXiv e-prints, arXiv:1706.06585

van Zee, L., \& Haynes, M. P. 2006, ApJ, 636, 214

Walter, F., Bolatto, A. D., Leroy, A. K., et al. 2017, ApJ, 835,265

Walterbos, R. A. M. 1991, in IAU Symposium, Vol. 144, The Interstellar Disk-Halo Connection in Galaxies, ed. H. Bloemen, 223-232

Wang, J., Hammer, F., Puech, M., Yang, Y., \& Flores, H. 2015, MNRAS, 452, 3551

Werk, J. K., Putman, M. E., Meurer, G. R., et al. 2008, ApJ, 678, 888

Wu, B., Tan, J. C., Nakamura, F., et al. 2017, ApJ, 835, 137

Zschaechner, L. K., \& Rand, R. J. 2015, ApJ, 808, 153 